\title{
Pairing and shell evolution in neutron rich nuclei
}

\author{
M. Saha Sarkar \\ Nuclear Physics Division, Saha Institute of Nuclear Physics, Kolkata 700064, INDIA \\ S. Sarkar \\ Department of Physics, Bengal Engineering and Science University, Shibpur, Howrah - 111103, INDIA
}

(Dated: November 16, 2018)

\begin{abstract}
From the experimental data on odd-even staggering of masses, we have shown that variation of pairing as a function of neutron number plays an important role in many distinctive features like occurrence of new shell closures, shell erosion, anomalous reduction of the energy of the first $2^{+}$ state and slower increase in the $\mathrm{B}\left(E 2,2_{1}^{+} \rightarrow 0_{1}^{+}\right)$in the neutron-rich even-even nuclei in different mass regions. New predictions have been made in a model independent way. Results of theoretical calculations support the phenomenological findings.
\end{abstract}

PACS numbers: 21.60.Cs,21.30.Fe,23.20.Lv,27.60.+j

The energy of the first $2^{+}$state $\left(E\left(2_{1}^{+}\right)\right)$of even-even nucleus is a sensitive probe to study the evolution of shell structure. For spherical nuclei, this state is formed by breaking a pair spending the pair binding energy, showing pronounced maxima at the shell closures. Nuclei away from the shell closures are gradually driven away from spherical symmetry, where "anomalously" low first excited collective $2^{+}$states are observed.

Usually, a few distinctive features have been identified as the signatures of changing shell structure away from stability [1]. Three new doubly magic Oxygen isotopes have been observed. For neutron rich nuclei, semi-magic ones like ${ }_{12}^{32} M g_{20},{ }_{10}^{30} N e_{20}$ have shown erosion of $\mathrm{N}=20$ shell closure with sudden decrease in their $E\left(2_{1}^{+}\right)$values and an increase in their corresponding $\mathrm{B}\left(E 2,2_{1}^{+} \rightarrow 0_{1}^{+}\right)$. However some recent measurements of reduced $\left(E\left(2_{1}^{+}\right)\right)$ and $\mathrm{B}(\mathrm{E} 2)$ values in highly neutron nuclei having $\mathrm{Z}=4-18$ have raised a serious discussion on hindered E2 strength unexpected for these nuclei phenomenologically as well as theoretically [2 5]. In heavier nuclei, after observation [6] of reduction of both $E\left(2_{1}^{+}\right)$and $\mathrm{B}(\mathrm{E} 2)$ in ${ }^{136} T e$, Terasaki et al. 7], from QRPA calculations, traced the origin of this anomalous behaviour in ${ }^{136} \mathrm{Te}$ isotope to a reduced neutron pairing above the $\mathrm{N}=82$ magic gap. Around this period, new empirical interactions [8] proposed for neutron rich isotopes above the ${ }^{132} \mathrm{Sn}$ core and later those for neutron rich nuclei in the $s d-f p$ shell [9, 10] also included reduction of pairing matrix elements for better reproduction of data. So these studies indicate an important role of pairing in the evolution of the structure of exotic nuclei.

In this letter, we show from the neutron and proton pairing energies estimated from the differences in experimental binding energies in different mass regions, that pairing plays a crucial role in evolution of shell structure in atomic nuclei. For observed shell erosions at $\mathrm{N}=8$ in Be isotopes and at $\mathrm{N}=20$ in $\mathrm{Ne}, \mathrm{Mg}$ isotopes, relative enhancement of proton pairing along with weakening of neutron pairing have been found to be important. We have found a clear correlation among the occurrence of new shell closures, the reduction of $E\left(2_{1}^{+}\right)$and hindered

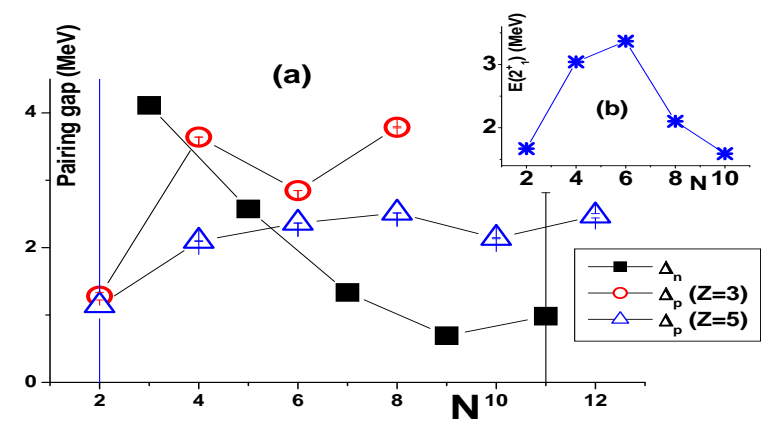

FIG. 1: Variation of (a) $\Delta_{n}$ for odd isotopes of Be, $\Delta_{p}$ for odd isotopes of $\mathrm{Li}$ and $\mathrm{B}$, (b) $E\left(2_{1}^{+}\right)$energies for even isotopes of $B e$ with increasing neutron number $(\mathrm{N})$.

$\mathrm{B}(\mathrm{E} 2)$ in the neutron-rich even-even nuclei and weakening of neutron pairing. This feature has been corroborated by Shell Model (SM) and Cranked Hartree Fock Bogoliubov (CHFB) results.

The empirical neutron and proton pairgaps are related to the odd-even staggering of binding energies [11]. They are defined as

$$
\begin{aligned}
\Delta_{n}(Z, N) & =\frac{\pi_{N}}{2}[B(Z, N-1)-2 * B(Z, N)+B(Z, N+1)] \\
\Delta_{p}(Z, N) & =\frac{\pi_{Z}}{2}[B(Z-1, N)-2 * B(Z, N)+B(Z+1, N)]
\end{aligned}
$$

where $\mathrm{B}(\mathrm{Z}, \mathrm{N})$ is the binding energy [12] of the nucleus with $\mathrm{Z}$ protons and $\mathrm{N}$ neutrons. The factor depending on the number parity $\pi_{N}\left(\pi_{Z}\right)$ is chosen so that the pairing gaps are all positive. To exclude the effect of variation of single particle energies in the staggering, the pairing gaps for odd N (Z)'s have been considered as the neutron (proton) pairing energies. The neutron (proton) pairing energy at an even $N(Z)$ is estimated from the average of corresponding values for two neighbouring odd $N(Z)$ nuclei.

In $\mathrm{Be}(\mathrm{Z}=4)$ isotopes, the $\mathrm{N}=8$ shell gap quenching has been observed [4]. Comparison of Figs. 1 $\mathrm{a}$ and $\mathrm{b}$ reveals how $2_{1}^{+}$states are built in Be isotopes as $\mathrm{N}$ increases. In ${ }^{6} B e$, this state is built by breaking a weak $\pi 1 p_{3 / 2}$ proton pair. In ${ }^{8,10} \mathrm{Be}$, both protons and neutrons 


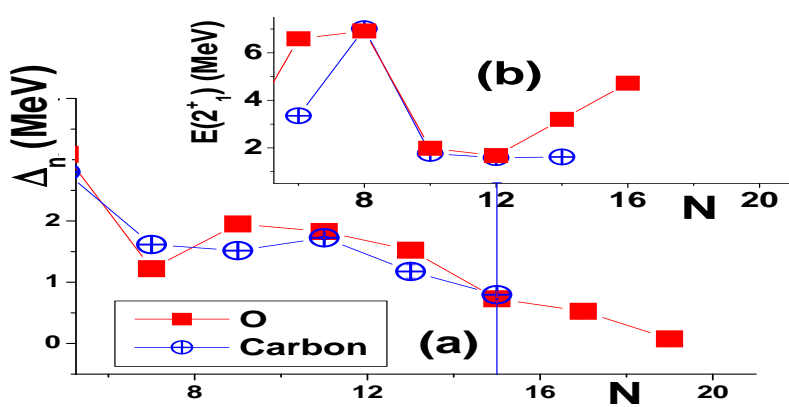

FIG. 2: Variation of (a) $\Delta_{n}$ for odd isotopes, (b) $E\left(2_{1}^{+}\right)$energies for even isotopes, of $C$ and $O$ with increasing neutron number $(\mathrm{N})$.

can contribute. The $2_{1}^{+}$state in ${ }^{10} \mathrm{Be}$ can be generated by breaking a neutron pair in $\nu 1 p_{3 / 2}$ and exciting one of them to $\pi 1 p_{1 / 2}$. As a result the $2_{1}^{+}$energy increases (Fig. 1b). With increasing neutron number, the neutron pairing decreases with increase in proton pairing. At around $\mathrm{N}=8$, the proton pairing becomes stronger (Fig. 17) by about $2 \mathrm{MeV}$. In ${ }^{12} \mathrm{Be}$, instead of breaking a strong proton pair $\left(\Delta_{p} \simeq 3.1 \mathrm{MeV}\right)$ in $\pi 1 p_{3 / 2}$ or exciting two neutrons across the $\mathrm{N}=8$ shell closure to $\nu 1 d_{5 / 2}$, the most favourable option to generate a $2^{+}$state, is to deform the system (nuclear Jahn Teller effect [13]) and bring down the energy of the $\nu 1 d_{5 / 2}$ orbit. The orbit below the gap, i.e the $1 / 2[101]$ Nilsson orbit originating primarily from $\nu 1 p_{1 / 2}$ at low deformations, is insensitive to increase in deformation compared to the downslope of the $1 / 2[220]$ orbit of $\nu 1 d_{5 / 2}$ [14]. This results in closing of the $\mathrm{N}=8$ shell gap for ${ }^{12} \mathrm{Be}$ isotope. The single particle energy (spe) difference $\nu\left(1 p_{1 / 2}-1 d_{5 / 2}\right)$ in $\mathrm{Be}$, at $\mathrm{N}=8$, estimated from difference of $\Delta_{n}$ of relevant even $\mathrm{N}$ and $(\mathrm{N}+1)$ [1] isotopes is $\approx 1.8 \mathrm{MeV}$ compared to $\approx 3.9 \mathrm{MeV}$ in corresponding $\mathrm{C}$ isotopes providing evidence of shell erosion. The weakening of the neutron pairing $\left(\Delta_{n} \simeq 0.8\right.$ $\mathrm{MeV}$ at $\mathrm{N}=10$ ) further decreases the $E\left(2_{1}^{+}\right)$energies of ${ }^{14} \mathrm{Be}$ without substantial increase in deformation [4].

For $\mathrm{C}$ and $\mathrm{O}$ isotopes at $\mathrm{N}=8$, the proton pairing is nearly equal to the neutron pairing. Fig. 2 a shows that the $\Delta_{n}$ 's for carbon and oxygen isotopes decrease with $\mathrm{N}$ above $\mathrm{N}=11$. For the closure of the neutron $1 d_{5 / 2}$ and $2 s_{1 / 2}$ subshells at $\mathrm{N}=14$ and 16 , the semi-magic oxygen isotopes exhibit shell closures at ${ }^{22,24} \mathrm{O}$, respectively (Fig. 2 2 ). The presence of two valence proton holes inhibits the occurrence of shell closure for the carbon isotopes. The spe difference $\nu\left(1 d_{5 / 2}-2 s_{1 / 2}\right)$ and $\Delta_{n}$ at $\mathrm{N}=14$, are $\simeq(2.7,1.1) \mathrm{MeV}$ and $(1.3,1.0) \mathrm{MeV}$ for $\mathrm{O}$ and $\mathrm{C}$ isotopes, respectively. In ${ }^{24} O, \nu\left(2 s_{1 / 2}-1 d_{3 / 2}\right)$ is $\simeq 3.9$ $\mathrm{MeV}$ compared to $\Delta_{n} \simeq 0.6 \mathrm{MeV}$. However, the weakening of pairing in carbon isotopes is manifested through decrease in its $\mathrm{E}\left(2_{1}^{+}\right)$values.

Another prominent example of the erosion of magicity is observed at $N=20$ for $Z=10,12$. In Fig. 3a, variation of $\Delta_{p}-\Delta_{n}(=\Delta)$, the difference between proton and neutron pairing energies with increasing $\mathrm{N}$ has been shown. For $\mathrm{Ne}$ and $\mathrm{Mg}$ isotopes, across $\mathrm{N}=20$, the proton pairing shows an increase with corresponding decrease in

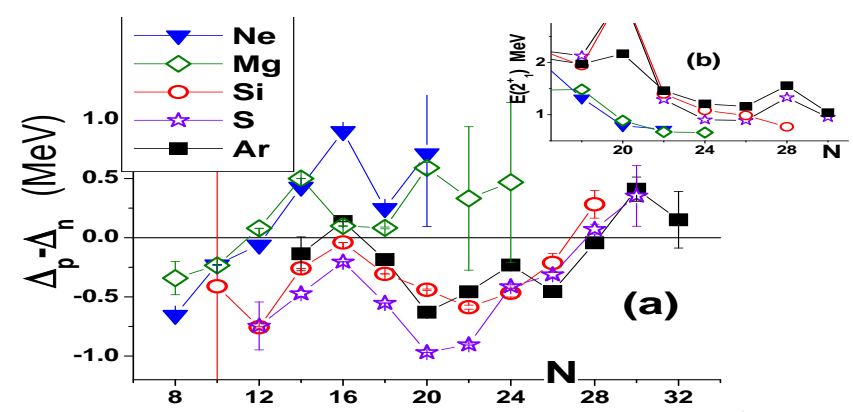

FIG. 3: Variation of (a) $\Delta_{p}-\Delta_{n}$ and (b) $E\left(2_{1}^{+}\right)$energies for even isotopes of $\mathrm{Ne}, \mathrm{Mg}, \mathrm{Si}, \mathrm{S}$ and $\mathrm{Ar}$ with increasing neutron number $(\mathrm{N})$.

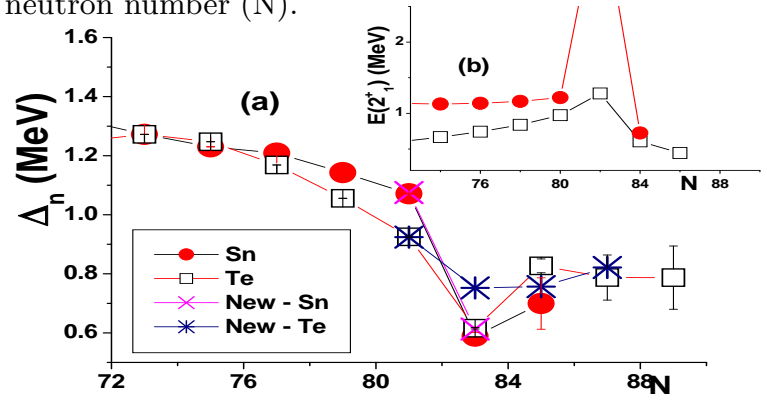

FIG. 4: Same as Fig. 2 for $S n$ and Te. "New" indicates the $\Delta_{n}$ calculated from most recent mass measurements [18].

neutron pairing ( $\Delta$ positive). The situation is similar to $\mathrm{Be}$ isotopes at $\mathrm{N}=8$. The Nilsson orbitals involved are upsloping $3 / 2[202]$ from $\nu 1 d_{3 / 2}$ and relatively faster downsloping $1 / 2[330]$ from $\nu 1 f_{7 / 2}$. So onset of deformation along with reduced neutron pairing depresses the $E\left(2_{1}^{+}\right)$in $\mathrm{N}=20$ isotopes of $\mathrm{Ne}$ and $\mathrm{Mg}$ without substantial increase in $\mathrm{B}(\mathrm{E} 2) \mathrm{s}$. The weakening of the neutron pairing continues for higher $\mathrm{N}$ with further decrease in the $E\left(2_{1}^{+}\right)$energies of ${ }^{32} \mathrm{Ne},{ }^{34} \mathrm{Mg}$. For Si, S and $\mathrm{Ar} n u-$ clei, below $\mathrm{N}=28, \Delta$ is negative (except for $\mathrm{Ar}$ at $\mathrm{N}=16$ ), indicating stronger neutron pairing. However, in Si nuclei, $\Delta$ crosses the zero line and becomes positive from $\mathrm{N} \simeq 27$, while in $\mathrm{S}$ and $\mathrm{Ar}$ crossing is at $\mathrm{N}=28$. So Si shows an erosion of the $\mathrm{N}=28$ shell closure manifested through depression in $\mathrm{E}\left(2_{1}^{+}\right)$energy (Fig. 3b) due to onset of deformation where neutrons from upsloping $7 / 2$ [303] from $\nu 1 f_{7 / 2}$ can be excited to downsloping $1 / 2[321]$ of $\nu 2 p_{3 / 2}$, with $5 / 2[312]$ from $\nu 1 f_{7 / 2}$ being insensitive to increase in deformation [14]. In $\mathrm{S}$ and Ar isotopes, erosion is not distinct, but beyond $\mathrm{N}=28$, depression in $\mathrm{E}\left(2_{1}^{+}\right)$with slower increase in B(E2) is observed [5].

The Sn region is even more interesting, showing how new features develop as one moves far away from stabilty for heavy nuclei. For neutron-rich isotopes of Sn and other heavier nuclei, neutron pairing shows reduction. However, the proton pairing is generally smaller than or close to neutron pairing for these nuclei. The region above neutron-rich doubly closed ${ }^{132} \mathrm{Sn}$ has great similarities [15] with that above doubly closed ${ }^{208} \mathrm{~Pb}$ on the stability. The even $S n$ isotopes have nearly constant $E\left(2_{1}^{+}\right)(\simeq 1200 \mathrm{keV})$ values for $\mathrm{A}=102-130$. This is attributed to the near degeneracy of the neutron $1 g_{7 / 2}$ and $2 d_{5 / 2}$ single particle orbitals which enhances pairing correlations. Similarly, there is a general belief that for $\mathrm{Sn}$ 
isotopes beyond ${ }^{132} \mathrm{Sn}$, there will be enhancement of pairing resulting in a constant $0_{1}^{+}-2_{1}^{+}$spacing, as revealed in calculations with realistic interactions [16, 17]. However, suddenly at $\mathrm{N}=84$, for ${ }^{134} S n$, the $E\left(2_{1}^{+}\right)$decreases

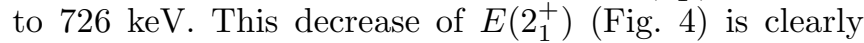
correlated to the decrease in pairing manifested through the reduction in $\Delta_{n}(\simeq 1.2 \mathrm{MeV}$ at $\mathrm{N}=81$ to $0.6 \mathrm{MeV}$ at $\mathrm{N}=83$ ). This observation is strengthened by the most recent precise mass measurements [18]. On the other hand, for $\mathrm{Pb}$ isotopes above $\mathrm{N}=126$, the $\Delta_{n}$ values are very similar to those below $\mathrm{N}=126(\simeq 0.68,0.32,0.62 \mathrm{MeV}$ at $\mathrm{N}=123,125,127$, respectively) and so a near constancy of the $E\left(2_{1}^{+}\right)$s below and above $\mathrm{N}=126$ closure $(0.899,0.803$, $0.800 \mathrm{MeV}$ for $\mathrm{N}=122,124,128$, respectively) has been observed. Pairing for different isotopes of Te, Xe and Ba nuclei has been estimated. At $\mathrm{N}=82, E\left(2_{1}^{+}\right)(1.279$, 1.313 and $1.435 \mathrm{MeV}$, respectively) for $\mathrm{Te}$, Xe and $\mathrm{Ba}$ is $\simeq 2 \Delta_{p}\left(\Delta_{p} \simeq 0.66,0.82\right.$ and $0.87 \mathrm{MeV}$, respectively). Compared to that in $\mathrm{N}=78\left(\Delta_{n} \simeq 1.1 \mathrm{MeV}\right.$ for Te, Xe and $\mathrm{Ba})$, beyond $\mathrm{N}=82$, reduction in neutron pairing $\left(\Delta_{n}\right.$ $\simeq 0.72,0.83,0.84 \mathrm{MeV}$ at $\mathrm{N}=84$ for $\mathrm{Te}, \mathrm{Xe}$ and $\mathrm{Ba}$, respectively) is observed. It gives rise to depressed $E\left(2_{1}^{+}\right)$ (0.606, 0.589 and $0.602 \mathrm{MeV}$, respectively) and relatively hindered $\mathrm{B}(\mathrm{E} 2) \mathrm{s}$ depending on the degree of reduction. Weaker $\Delta_{p}$ at $\mathrm{N}=82$ favour easy generation of $2_{1}^{+}$state without any need for neutron shell erosion.

Analysing the empirical observations of the features of neutron-rich nuclei one can frame the following rules.

- For proton - magic nuclei, reduction in neutron pairing will give rise to

- anomalous decrease in $\mathrm{E}\left(2_{1}^{+}\right)$with slower increase in deformation for isotopes with neutrons in unfilled subshells. For normal pairing observed near stability, similar value of low $\mathrm{E}\left(2_{1}^{+}\right)$'s will indicate higher value of $\mathrm{B}(\mathrm{E} 2)$ or larger deformation.

- appearance of new shell closures manifested through enhancement of $\mathrm{E}\left(2_{1}^{+}\right)$for isotopes with filled up sub-shells. The energy gap between the sub - shells must be substantially greater than the neutron pairing energy.

- For nuclei with a few valence particles near shell closure having unfilled proton sub-shell, reduced neutron pairing will lead to

- anomalous decrease in $\mathrm{E}\left(2_{1}^{+}\right)$with slower increase in deformation for isotopes with neutrons in unfilled sub-shells,

- onset of deformation for $\mathrm{N}_{\text {magic }}$ nucleus, resulting in erosion of shell gap if proton pairing shows strong enhancement. This will be usually observed for lighter mass nuclei.

To have a quantitative estimation of the effect of pairing on depression in the $E\left(2_{1}^{+}\right)$values and on the onset of deformation, shell model calculation has been done for oxygen isotopes. It has been shown that with reduction of $0^{+}$diagonal two body matrix elements of Bonn A realistic interaction [19], $E\left(2_{1}^{+}\right)$energies of ${ }^{18-22} O$ decreases
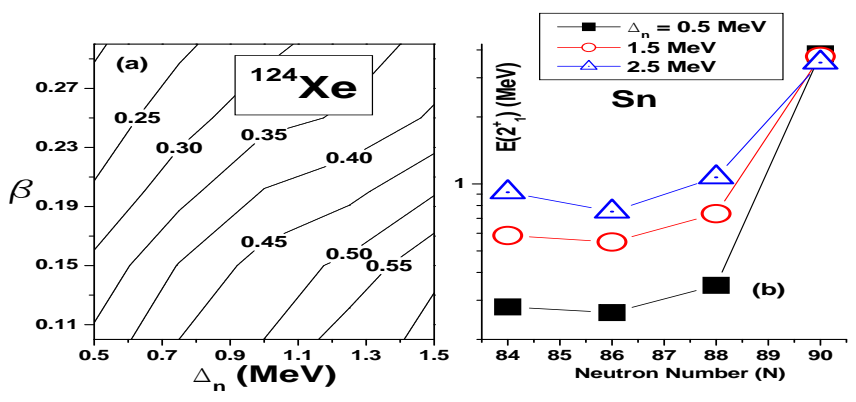

FIG. 5: (a) The equi- $\mathrm{E}\left(2_{1}^{+}\right)$energy contours are shown as a function of deformation $(\beta)$ and neutron pairing gap $\left(\Delta_{n}\right)$. (b) The variations in $\mathrm{E}\left(2_{1}^{+}\right)$energies for ${ }^{134-140} \mathrm{Sn}$ with reduction in neutron pairing gap.

without any increase in the $\mathrm{B}(\mathrm{E} 2)$ values with improvement in prediction of a shell gap at $\mathrm{N}=14$, usually not predicted by Bonn-A. The same is also true for even-even Sn isotopes beyond ${ }^{132} \mathrm{Sn}$ as also discussed in Ref. [16].

From cranked Hartree-Fock-Bogoliubov (CHFB) calculations with a pairing plus quadrupole Hamiltonian, equi-energy contours for $E\left(2_{1}^{+}\right)$energies in ${ }^{124} \mathrm{Xe}$, well studied within this formalism [20] have been plotted in Fig. $5 \mathrm{k}$, for variations of neutron pairing gap and quadrupole deformation. For all these contours the proton pairing gap has been kept constant at $1.5 \mathrm{MeV}$, which is obtained from the experimental odd-even mass difference. These contours are useful to understand the effect of variation of pairing on $\mathrm{E}\left(2_{1}^{+}\right)$for fixed value of deformation and vice-versa. For example, the measured $\mathrm{E}\left(2_{1}^{+}\right)$ value $(\simeq 350 \mathrm{keV})$ for ${ }^{124} \mathrm{Xe}$ can correspond to two extreme combinations of neutron pairing gap and deformation. They are $\left(\Delta_{n}=1.4\right.$ and $\left.\beta=0.30\right)$ and $\left(\Delta_{n}=0.5\right.$ and $\beta=0.11$ ) (Fig. 5 ) ). It implies that same depressed value of energy can result either due to increase in deformation with usual pairing or relatively smaller deformation with reduced of pairing.

Self-consistent solutions have been obtained to investigate how deformation changes with decreasing pairing and vice - versa for ${ }^{124} \mathrm{Xe}$. The results are shown in Table I. It is found that even without any change of quadrupole interaction strength, reduction in neutron pairing strength favours a slow onset of deformation (Part (a) of Table I). It is also evident that same depressed $\mathrm{E}\left(2_{1}^{+}\right)$energy (e.g., $\left.\simeq 120-150 \mathrm{keV}\right)$ can result either from a reduced pairing $\left(\Delta_{n} \simeq 0\right)$ and relatively smaller deformation $\left(\beta_{2} \simeq 0.26\right)$ (Table Ia), or from enhanced deformation $\left(\beta_{2} \simeq 0.37\right)$ for stronger pairing $\left(\Delta_{n} \simeq 0.6 \mathrm{MeV}\right)$ (Table Ib).

To test the observation for proton-magic nuclei, we have done another set of calculations for $S n$ isotopes above ${ }^{132} \mathrm{Sn}$. Single particle energies have been obtained from shell model Hamiltonian in Refs. [8, 16, 21]. The single particle energies for the $\pi 1 g_{9 / 2}$ and $\nu 1 h_{11 / 2}$ orbitals have been chosen with sufficient care so that $S n$ isotopes above $A=132$ do not show any protons or neutron excitations across the $\mathrm{Z}=50$ and $\mathrm{N}=82$ shell closures, respectively. The remaining single particle energies are calcu- 
TABLE I: Effects of (a) decrease in neutron pairing strength $\left(\mathrm{g}_{n}\right)$ and $(\mathrm{b})$ increase in quadrupole interaction strength, $x_{2}$, on ground state deformation, pairing gaps and $\mathrm{E}\left(2_{1}^{+}\right)$values of ${ }^{124} \mathrm{Xe}$. In part (a), the $x_{2}$ value is kept constant at 72.0 , in (b), the value of $g_{n}$ is 20.0. The proton pairing strength $\left(g_{p}\right)$ is kept constant at 26.0 in all these calculations. The energies are in $\mathrm{MeV}$.

\begin{tabular}{cccccc}
\hline \hline & $g_{n}$ & $\beta_{2}$ & $\Delta_{p}$ & $\Delta_{n}$ & $\mathrm{E}\left(2_{1}^{+}\right)$ \\
\hline$(a)$ & 25 & 0.087 & 1.368 & 1.875 & 0.773 \\
& 20 & 0.182 & 1.222 & 1.047 & 0.304 \\
& 15 & 0.230 & 1.171 & 0.433 & 0.172 \\
& 10 & 0.261 & 1.168 & 0.00003 & 0.125 \\
& 5 & 0.261 & 1.168 & 0.00001 & 0.114 \\
\hline & $x_{2}$ & $\beta_{2}$ & $\Delta_{p}$ & $\Delta_{n}$ & $\mathrm{E}\left(2_{1}^{+}\right)$ \\
\hline$(b)$ & 72 & 0.182 & 1.222 & 1.047 & 0.304 \\
& 77 & 0.256 & 1.167 & 0.834 & 0.233 \\
& 82 & 0.366 & 1.116 & 0.605 & 0.151 \\
\hline \hline
\end{tabular}

lated using Nilsson prescription [22] with proper normalisation.

From the Fig. 5b, it is observed that as pairing energy for neutron decreases, the shell closure at N=90 [21] manifested through sudden jump in $\mathrm{E}\left(2_{1}^{+}\right)$energy of ${ }^{140} \mathrm{Sn}$ compared to that in ${ }^{138} \mathrm{Sn}$ becomes more pronounced. For stronger pairing the $\mathrm{E}\left(2_{1}^{+}\right)$energies of semi-magic nuclei show much slower variation with increasing neutron numbers, as also observed for isotopes of Sn below
${ }^{132} \mathrm{Sn}$.

From the experimental data on odd-even staggering of masses and SM and CHFB calculations, we have shown that pairing plays an important role in the shell evolution of neutron-rich nuclei. We have identified a few distinctive features of neutron-rich nuclei based on our analysis of the empirical data. For proton-magic nuclei, due to reduction of neutron pairing away from stability, each sub-shell closure will be manifested as a new shell closure, if energy gap between sub-shells is greater than $\Delta_{n}$. For other neutron-rich isotopes for which proton numbers are not magic, the $\mathrm{E}\left(2_{1}^{+}\right)$energies at each existing neutron shell closure will show dramatic decrease depending on the extent of reduction in neutron pairing. This reduction will also be manifested through slow onset of deformation at shell closures. But these values of the deformation will not correspond to the same variation as seen on the stability. The increase in deformation with decrease in energy of the $2_{1}^{+}$state will be much slower for reduced pairing. For lighter mass nuclei, increase in proton pairing has been found to play a significant role in the observed shell erosion. This work therefore conclusively shows the role of variation in pairing as a function $\mathrm{N}$ in nuclear shell evolution of neutron - rich nuclei. This method of analysis is also applicable to exotic proton-rich nuclei. The microscopic origin of variation in pairing with $\mathrm{N}$ constitutes a separate important issue. Our work also warrants more precise measurement of masses away from stability and analysis of mass data to search for newer isotopes for which such shell evolution can be expected.
[1] J. P. Schiffer et al., Phys. Rev. Lett. 92, 162501 (2004); T. Otsuka, T. Suzuki, R. Fujimoto, H. Grawe, Y. Akaishi, ibid, 95, 232502 (2005); T. Otsuka, T. Suzuki, J.D. Holt, A. Schwenk, Y. Akaishi, ibid, 105, 032501 (2010); O. Sorlin, M. G. Porquet, Prog. Part. and Nucl. Phys. 61, 602 (2008).

[2] S. Raman, C.W. Nestor, Jr., P. Tikkanen, At. Data Nucl. Data Tables 78, 1 (2001); http://www.nndc.bnl.gov.

[3] M. Petri et al., Phys. Rev. Lett. 107, 102501 (2011); N. Imai, et al., ibid, 92062501 (2004); H.J. Ong et al., Eur. Phys. J. A 42, 393 (2009); A. Umeya et al., Nucl. Phys. A 829, 13 (2009).

[4] N. Aoi et al., Phys. Rev. C 66, 014301 (2002); T. Sugimoto et al., Phys. Lett. B 654, 160 (2007).

[5] R. Winkler et al., arXiv:1203.4630v1 [nucl-ex] 21 Mar 2012.

[6] D. C. Radford et al., Phys. Rev. Lett. 88, 222501 (2002).

[7] J. Terasaki, J. Engel, W. Nazarewicz, and M. Stoitsov, Phys. Rev. C 66, 054313 (2002).

[8] Sukhendusekhar Sarkar, Proc. DAE Symp. Nucl. Phys. (India) A 45, 72 (2002); Sukhendusekhar Sarkar, M. Saha Sarkar, Eur. Phys. Jour. A21, 61 (2004).

[9] Angelo Signoracci, B. A. Brown, and Morten HjorthJensen, Phys. Rev. C 83, 024315 (2011)

[10] F. Nowacki and A. Poves, Phys. Rev. C 79, 014310 (2009).

[11] W. Satula, J. Dobaczewski, W. Nazarewicz, Phys. Rev.
Lett. 81, 3599 (1998); W.A. Friedman and G.F. Bertsch, Eur. Phys. J. A 41, 109 (2009).

[12] Georges Audi and Wang Meng, Private Communication April 2011.

[13] P.G. Reinhard, E.W. Otten, Nucl. Phys. A 420, 173 (1984); W. Nazarewicz, Nucl. Phys. A 574, 27 (1994).

[14] Ikuko Hamamoto, Phys. Rev. C 76, 054319 (2007); http://ie.lbl.gov/toipdf/nilsson.pdf

[15] J. Blomqvist, Proceedings of the 4th International Conference on Nuclei Far from Stability, Denmark, 1981 (CERN, Geneva, 1981), p. 536.

[16] S. Sarkar and M. Saha Sarkar, Phys. Rev. C 78, 024308 (2008).

[17] M. P. Kartamyshev, T. Engeland, M. Hjorth-Jensen, and E. Osnes, Phys. Rev. C 76, 024313 (2007).

[18] J. Hakala et al., arXiv:1203.0958v2 [nucl-ex] 6 Mar 2012; J. Van Schelt et al., arXiv:1203.4470v1 [nucl-ex] 20 Mar 2012.

[19] Morten Hjorth-Jensen, Thomas T.S. Kuo, Eivind Osnes, Phys. Rep. 261, 125 ( 1995).

[20] M. Saha Sarkar and S.Sen, Phys. Rev. C 56, 3140 (1997) and references therein.

[21] S. Sarkar and M. Saha Sarkar, Phys. Rev. C 81, 064328 (2010).

[22] S.G. Nilsson et al., Nucl. Phys. A 131, 1 (1969). 